\title{
Pseudo-Schwarzschild description of transonic spherical accretion onto compact objects
}

\author{
Tapas K. Das ${ }^{1}$ and A. Sarkar ${ }^{2}$ \\ 1 Inter University Centre For Astronomy And Astrophysics, Post Bag 4 Ganeshkhind, Pune 411 007, India \\ e-mail: tapas@iucaa.ernet.in \\ 2 National Centre For Radio Astrophysics, TIFR, Post Bag 3 Ganeshkhind, Pune 411 007, India \\ e-mail: sarkar@ncra.tifr.res.in
}

Received 6 March 2001/ Accepted 9 May 2001

\begin{abstract}
A number of "modified" Newtonian potentials of various forms are available in the literature which accurately approximate some general relativistic effects important for studying accretion discs around a Schwarzschild black hole. Such potentials may be called "pseudo-Schwarzschild" potentials because they nicely mimic the spacetime around a non-rotating/slowly rotating compact object. In this paper, we examine the validity of the application of some of these potentials to study the spherically symmetric, transonic, hydrodynamic accretion onto a Schwarzschild black hole. By comparing the values of various dynamical and thermodynamic accretion parameters obtained for flows using these potentials with full general relativistic calculations, we have shown that though the potentials discussed in this paper were originally proposed to mimic the relativistic effects manifested in disc accretion, it is quite reasonable to use most of the potentials in studying various dynamical as well as thermodynamic quantities for spherical accretion to compromise between the ease of handling of a Newtonian description of gravity and the realistic situations described by complicated general relativistic calculations. Also we have shown that depending on the chosen regions of parameter space spanned by specific energy $\mathcal{E}$ and adiabatic index $\gamma$ of the flow, one potential may have more importance than another and we could identify which potential is the best approximation for full general relativistic flow in Scwarzschild space-time for particular values of $\mathcal{E}$ and $\gamma$.
\end{abstract}

Key words. accretion, accretion discs - black hole physics - hydrodynamics

\section{Introduction}

Stationary, spherically symmetric and transonic hydrodynamic accretion of adiabatic fluid on to a gravitating astrophysical object at rest was studied in a seminal paper by Bondi (1952) using purely Newtonian Potential and by including the pressure effect of the accreting material. Later, Michel (1972) discussed fully general relativistic polytropic accretion on to a Schwarzschild black hole by formulating the governing equations for steady spherical flow of a perfect fluid in the Schwarzschild metric. Following Michel's relativistic generalization of Bondi's treatment, Begelman (1978) discussed some aspects of the critical (sonic) points of the flow for such an accretion. Using an unrelaxed mono-energetic particle distribution and assuming the fact that the relaxation time of such a particle distribution is very long compared to the typical flow time scale or dynamical time scale of steady accretion on to black holes, Blumenthal \& Mathews (1976) developed a model where the connection between the nonrela-

Send offprint requests to: Tapas K. Das,

e-mail: tapas@iucaa.ernet.in tivistic to the relativistic regime of the spherically accreting material could be established. Taking the fully ionized one-temperature $\left(T_{\text {electron }}=T_{\text {proton }}\right)$ hydrogen gas (governed by an exact relativistic equation of state) to be the fundamental constituent of the accreting material, Brinkmann (1980) treated spherically symmetric stationary accretion in Schwarzschild space time and showed that the temperature of accreting material at the Schwarzschild radius is one order of magnitude smaller than the flow temperature obtained by using a simple polytropic equation of state. Recently, Malec (1999) provided the solution for general relativistic spherical accretion with and without back reaction and showed that relativistic effects enhance mass accretion when back reaction is neglected.

Meanwhile, the theory of the accretion disc found prior importance because of the fact that disc accretion describes more realistic astrophysical situations found in nature. The beginning of modern accretion disc physics is traditionally attributed to the two classical articles by Shakura \& Sunyaev (1973) and Novikov \& Thorne (1973). While Shakura \& Sunyaev (1973) calculated the disk structure and related phenomena using purely Newtonian 
potential, Novikov \& Thorne provided a fully general relativistic description of accretion discs around black holes; later on, some aspects of which were modified by Riffert \& Herold (1995). However, rigorous investigation of transonic disc structure was found to be extremely complicated in full general relativistic space time (Chakrabarti 1996 and references therein). At the same time it was understood that as relativistic effects play an important role in the regions close to the accreting black hole (where most of the gravitational potential energy is released), purely Newtonian gravitational potential (in the form $\Phi_{\text {Newton }}=-\frac{G M}{r}$ ) cannot be a realistic choice to describe transonic black hole accretion in general. To compromise between the ease of handling of a Newtonian description of gravity and the realistic situations described by complicated general relativistic calculations, a series of "modified" Newtonian potentials have been introduced to describe the general relativistic effects that are most important for accretion disk structure around Schwarzschild and Kerr black holes (see Artemova et al. 1996 for further discussion). Introduction of such potentials allows one to investigate the complicated physical processes taking place in disc accretion in a semi-Newtonian framework by avoiding pure general relativistic calculations that most of the features of spacetime around a compact object are retained and some crucial properties of the analogous relativistic solutions of disc structure could be reproduced with high accuracy. Hence, those potentials might be designated as "pseudo-Kerr" or "pseudo-Schwarzschild" potentials, depending on whether they are used to mimic the space time around a rapidly rotating or non rotating/slowly rotating (Kerr parameter $a \sim 0$ ) black hole respectively.

It is important to note that although a number of such "pseudo" potentials are available in the literature to study various aspects of disc accretion, no such potentials are available which had been solely derived to describe spherically symmetric accretion on to a Schwarzschild (or Kerr) black hole. In this paper, we will concentrate on some of the "pseudo-Schwarzschild" disc potentials (potentials introduced to study accretion discs around a Schwarzschild black hole) to investigate whether those potentials could be used to study Spherical accretion, and if so, how "good" the choice would be for various such potentials. Also, we would like to check which potential among those would be the "best-fit" to approximate the full general relativistic description of transonic, spherically symmetric accretion on to a Schwarzschild black hole. In doing so, we solve the equations of motion of spherically accreting fluid in full Schwarzschild space-time as well as for motion under various "pseudo"-potentials, to study the variation of different dynamical and thermodynamic quantities (like Mach number of the flow, flow temperature etc.) with radial distance measured from the accreting black hole for the full general relativistic spherical flow (hereafter FGRSF) as well as for accretion using various "pseudoSchwarzschild" potentials. We then compare the results obtained using such potentials with the solutions of exact relativistic problems in a Schwarzschild metric. The plan of the paper is as follows: In next section, we will describe four "pseudo-Schwarzschild" disc potentials available in the literature and some of their basic features. In Sect. 3, we will provide the basic equations governing spherically symmetric accretion in full relativistic as well as in various "pseudo"-relativistic spacetimes. In Sect. 4 we will discuss how to solve those equations to find various dynamical quantities which are to be mutually compared and we present our results. Finally in Sect. 5 we conclude by discussing the suitability of various "pseudo" potentials in approximating the results obtained from exact relativistic calculations. For the rest of this paper, we will use the terms "modified-Newtonian potential" and "pseudo (Schwarzschild) potentials" synonymously.

\section{Some basic features of various "pseudo-Schwarzschild" potentials}

From now, we will define the Schwarzschild radius $r_{\mathrm{g}}$ as $r_{\mathrm{g}}=\frac{2 G M_{\mathrm{BH}}}{c^{2}}$

(where $M_{\mathrm{BH}}$ is the mass of the black hole, $G$ is universal gravitational constant and $c$ is velocity of light in vacuum) so that the marginally bound circular orbit $r_{\mathrm{b}}$ and the last stable circular orbit $r_{\mathrm{s}}$ take the values $2 r_{\mathrm{g}}$ and $3 r_{\mathrm{g}}$ respectively for a typical Schwarzschild black hole. Also, total mechanical energy per unit mass on $r_{\mathrm{s}}$ (sometimes called "efficiency" e) may be computed as -0.057 for this case. Also, we will use a simplified geometric unit throughout this paper where radial distance $r$ is be scaled in units of $r_{\mathrm{g}}$, radial dynamical velocity $u$ and polytropic sound speed $a$ of the flow is scaled in units of $c$ (the velocity of light in vacuum), mass $m$ is scaled in units of $M_{\mathrm{BH}}$ and all other derived quantities would be scaled accordingly. Also, for simplicity, we will use $G=c=M=1$. Below we would like to briefly describe four different "pseudo-Schwarzschild" potentials (expressed in the system of units discussed above) and to provide the "free fall" acceleration obtained using such potentials in compact form.

Paczyński \& Wiita (1980) introduced a "pseudoSchwarzschild" potential of the form

$\Phi_{1}=-\frac{1}{2(r-1)}$

which accurately reproduces the positions of $r_{\mathrm{s}}$ and $r_{\mathrm{b}}$ and gives the value of efficiency to be -0.0625 . Also the Keplerian distribution of angular momentum obtained using this potential is exactly same as that obtained in pure Schwarzschild geometry. Although this potential (as well as the other "pseudo" potentials available in the literature) does not satisfy the boundary condition exactly on the horizon, however, close to the horizon, accretion flows are supposed to be highly supersonic and the dynamical infall time scale becomes too small to allow significant radiation from infalling fluid. Even if there are significant 


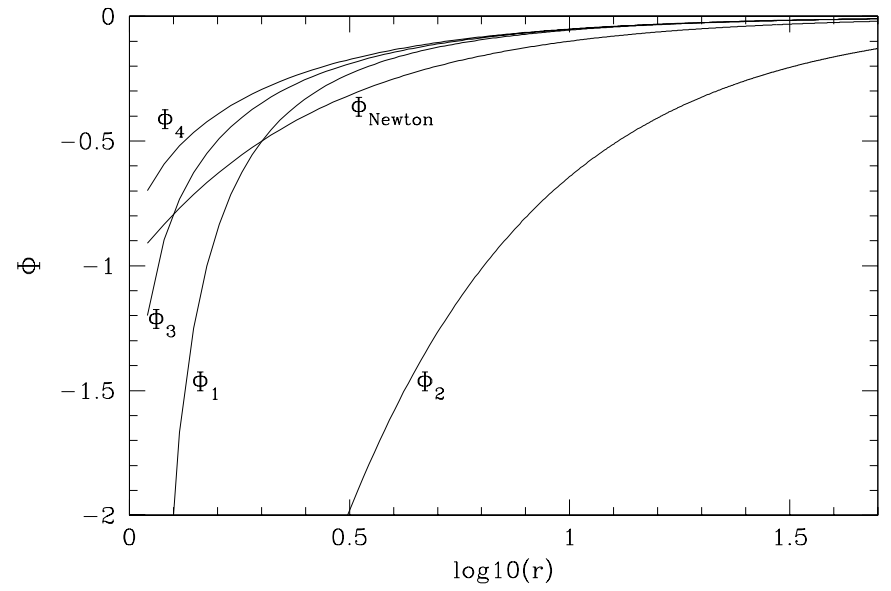

Fig. 1. Newtonian potential $\Phi_{\text {Newton }}(r)$ and other pseudopotentials $\Phi_{i}(r)$ 's $(i=1,2,3,4)$ are plotted as a function of the logarithmic radial distance from the accreting black hole. Except $\Phi_{2}$, all other pseudo-potentials have a singularity at one Schwarzschild radius $r_{\mathrm{g}}$. For $r>2 r_{\mathrm{g}}$, while the relative stiffness factor $\mathcal{S}$ is maximum for $\Phi_{4}$, it is minimum for $\Phi_{2}$, see text for details.

amounts of radiation, it does not contribute too much to the overall radiation coming out from the disc, due to the fact that radiation emitted from the very close viscinity of the black hole is highly redshifted. Thus, errors in calculation close to the horizon may have very little impact on the emitted disc spectrum and for all practical purposes, the Paczyński \& Wiita (1980) potential is considered to be the best approximation of pure Schwarzschild spacetime while treating the disc accretion (Artemova 1996). It is worth mentioning here that this potential was first introduced to study a thick accretion disc with super Eddington Luminosity. Also, it is interesting to note that although it had been thought of in terms of disc accretion, it is spherically symmetric (with a scale shift of $r_{\mathrm{g}}$ ). So in principle, it can definitely be used to study spherical accretion also on to a Schwarzschild black hole.

To analyse the normal modes of accoustic oscillations within a thin accretion disc around a compact object (slowly rotating black hole or weakly magnetized neutron star), Nowak \& Wagoner (1991) approximated some of the dominant relativistic effects of the accreting black hole (slowly rotating or nonrotating) via a modified Newtonian potential of the form

$\Phi_{2}=-\frac{1}{2 r}\left[1-\frac{3}{2 r}+12\left(\frac{1}{2 r}\right)^{2}\right]$

$\Phi_{2}$ has correct form of $r_{\mathrm{s}}$ as in the Schwarzschild case but is unable to reproduce the value of $r_{\mathrm{b}}$. This potential has the correct general relativistic value of the angular velocity (as measured at infinity) at $r_{\mathrm{s}}$. Also it reproduces the radial epicyclic frequency $\kappa$ (for $r>r_{\mathrm{s}}$ ) close to its value obtained from general relativistic calculations. However, this potential gives the value of efficiency as -0.064 which is larger than that produced by $\Phi_{1}$, hence the disc spec- trum computed using $\Phi_{2}$ would be more luminous compared to a disc structure studied using $\Phi_{1}$.

Remembering that the free-fall acceleration plays a very crucial role in Newtonian gravity, Artemova et al. (1996) proposed two different "pseudo" potentials to study disc accretion around a non-rotating black hole. The first potential proposed by them produces exactly the same value of the free-fall acceleration of a test particle at a given value of $r$ as is obtained for a test particle at rest with respect to the Schwarzschild reference frame, and is given by

$\Phi_{3}=-1+\left(1-\frac{1}{r}\right)^{\frac{1}{2}}$.

The second one gives the value of the free fall acceleration that is equal to the value of the covariant component of the three dimensional free-fall acceleration vector of a test particle that is at rest in the Schwarzschild reference frame and is given by

$\Phi_{4}=\frac{1}{2} \ln \left(1-\frac{1}{r}\right)$.

Efficiencies produced by $\Phi_{3}$ and $\Phi_{4}$ are -0.081 and -0.078 respectively. The magnitude of efficiency produced by $\Phi_{3}$ being maximum, calculation of disc structure using $\Phi_{3}$ will give the maximum amount of energy dissipation and the corresponding spectrum would be the most luminous one. However, as both $\Phi_{3}$ and $\Phi_{4}$ stems from the consideration of free fall acceleration and calculates the dependence of free fall accleration on radial distance in the Schwarzschild metric (which describes a spherically symmetric gravitational field in vacuum), it appears to be quite justified to use those potentials to study spherically symmetric accretion.

From now we will refer to all these four potentials as $\Phi_{i}$ in general where $\{i=1,2,3,4\}$ would correspond to $\Phi_{1}$ (Eq. (1a)), $\Phi_{2}$ (Eq. (1b)), $\Phi_{3}$ (Eq. (1c)) and $\Phi_{4}$ (Eq. (1d)) respectively. In Fig. 1, we plot various $\Phi_{i}$ 's as a function of the radial distance measured from the accreting black hole in units of $r_{\mathrm{g}}$. Also in the same plot, purely Newtonian potential $\Phi_{\text {Newton }}$ is plotted. If we now define a quantity $\mathcal{S}_{i}$ to be the "relative stiffness" of a potential $\Phi_{i}$ as:

$\mathcal{S}_{i}=\frac{\Phi_{i}}{r}$

(that is, $\mathcal{S}_{i}$ is a measure of the numerical value of any $i$ th potential at a radial distance $r$ ), we find that for $r>2 r_{\mathrm{g}}$ :

$\mathcal{S}_{2}<\mathcal{S}_{\text {Newton }}<\mathcal{S}_{1}<\mathcal{S}_{3}<\mathcal{S}_{4}$

which indicates that while $\Phi_{2}$ is a "flatter" potential compared to the pure Newtonian potential $\Phi_{\text {Newton }}$, all other "pseudo" potentials are "steeper" to $\Phi_{\text {Newton }}$ for $r>2 r_{\mathrm{g}}$.

One can write the modulus of free fall acceleration obtained from all "pseudo" potentials except for $\Phi_{2}$ in a compact form as

$\left|\Phi_{i}^{\prime}\right|=\frac{1}{2 r^{2-\delta_{i}}(r-1)^{\delta_{i}}}$ 
where $\delta_{1}=2, \delta_{3}=\frac{1}{2}$ and $\delta_{4}=1$. $\left|\Phi_{i}^{\prime}\right|$ denotes the absolute value of the space derivative of $\Phi_{i}$, i.e.,

$\left|\Phi_{i}^{\prime}\right|=\left|\frac{\mathrm{d} \Phi_{i}}{\mathrm{~d} r}\right|$

whereas acceleration produced by $\Phi_{2}$ can be computed as,

$\Phi_{2}^{\prime}=\frac{1}{2 r^{2}}\left(1-\frac{3}{r}+\frac{9}{2 r^{2}}\right)$.

In the next section,we would like to describe how one can investigate transonic spherical accretion using these potentials. Also, we will discuss how to calculate various dynamical quantities for full general relativistic bondi flow in a Schwarzschild metric. One standard method to investigate classical transonic bondi flow is to formulate the basic conservation equations, i.e., conservation of baryon number (obtained by integrating continuity equation) and conservation of specific energy (obtained by integrating Euler's equation using a specific equation of state for accreting matter) and then to simultaneously solve these conservation equations to get critical (sonic) quantities as functions of various accretion parameters (like specific energy $\mathcal{E}$, adiabatic index $\gamma$ or accretion rate $\dot{M}_{\text {in }}$ in the flow) and also to calculate the values of various dynamical and thermodynamic quantities (like Mach number $M$, of the flow, flow temperature, $T$, etc.) as functions of various accretion parameters (like $\mathcal{E}, \gamma, \dot{M}_{\text {in }}$ etc.) or radial distance (measured from the central accretor in the unit of Schwarzschild radius $r_{\mathrm{g}}$ ). Also a common practice is to study the variation of Mach number $M$ (ratio of the local dynamical velocity $u$ to the local sound velocity $a$; $M=\frac{u}{a}$ ) with radial distance $r$ (measured in units of $r_{\mathrm{g}}$ ) to investigate the "transonicity" of the flow for a fixed set of input parameters. We will perform the above mentioned calculations for accretion in pure Schwarzschild spacetime as well as for accretion in "pseudo-Schwarzschild" space time using $\Phi_{i}$ 's and compare the results obtained for various $\Phi_{i}$ 's with exact relativistic calculations. As accretion onto a black hole is necessarily transonic to satisfy the inner boundary condition at the event horizon, unlike Bondi's (1952) original work, here we will concentrate only on transonic solutions, i.e., we will deal with accretion (wind) which is subsonic (supersonic) far away from the black hole and approaches (moves away from) the hole supersonically (subsonically) after crossing a sonic point (the location of which can be determined as a function of $\mathcal{E}$ and $\gamma$, see Sect. 3 ) on its way towards (away from) the accretor.

\section{Governing equations}

\subsection{Full General Relativistic Spherical Flow (FGRSF) in Schwarzschild space-time}

For a schwarzschild metric of the form

$$
\mathrm{d} s^{2}=\mathrm{d} t^{2}\left(1-\frac{1}{r}\right)-\mathrm{d} r^{2}\left(1-\frac{1}{r}\right)^{-1}-r^{2}\left(\mathrm{~d} \theta^{2}+\sin ^{2} \theta \mathrm{d} \phi^{2}\right)
$$

the energy momentum tensor $T^{\alpha \beta}$ for a perfect fluid can be written as (Shapiro \& Teukolsky 1983)

$T^{\alpha \beta}=\epsilon u^{\alpha} u^{\beta}+p\left(u^{\alpha} u^{\beta}-g^{\alpha \beta}\right)$

where $\epsilon$ and $p$ are proper energy density and pressure of the fluid (evaluated in the local inertial rest frame of the fluid) respectively and $u^{\alpha}$ is the four velocity commonly known as

$u^{\alpha}=\frac{\mathrm{d} x^{\alpha}}{\mathrm{d} s}$.

Equations of motion which are to be solved for our purpose are,

1) Conservation of mass flux or baryon number conservation:

$\left(\rho u_{\alpha}\right)_{; \alpha}=0$

and

2) Conservation of momentum or energy flux (the general relativistic Euler equation obtained by taking the four divergence of $\left.T^{\alpha \beta}\right)$ :

$(\epsilon+p) u_{\alpha ; \beta} u^{\beta}=-p_{, \alpha}-u_{\alpha} p_{, \beta} u_{,}^{\beta}$

where the semicolons denote the covariant derivatives.

Following Michel (1972), one can rewrite Eqs. (3a) and $(3 \mathrm{~b})$ for spherical accretion as

$4 \pi \rho u r^{2}=\dot{M}_{\text {in }}$

and

$\left(\frac{p+\epsilon}{\rho}\right)^{2}\left(1-\frac{1}{r}+u^{2}\right)=C$

as two fundamental conservation equations for time independant hydrodynamical flow of matter on to a Schwarzschild black hole without back-reaction of the flow on to the metric itself. $\dot{M}_{\text {in }}$ is the mass accretion rate and $C$ is a constant (related to the total enthalpy influx) to be evaluated for a specific equation of state.

For a polytropic equation of state i.e.,

$p=K \rho^{\gamma}$

and defining $\dot{\mathcal{M}}=\dot{M}_{\text {in }} \gamma^{n} K^{n}$, where $\dot{\mathcal{M}}$ is a measure of the entropy of the flow ( $n$ is a polytropic constant of the flow defined as $\left.n=(\gamma-1)^{-1}\right)$ and is another conserved quantity of the flow, one can rewrite conservation Eqs. (4a) and (4b) as (see Chakrabarti 1996 and references therein):

$\mathcal{E}=1+h u_{\mathrm{t}}=1+\left(\frac{p+\epsilon}{\rho}\right)\left(\frac{1-\frac{1}{r}}{1-u^{2}}\right)^{\frac{1}{2}}$

and

$\dot{\mathcal{M}}=4 \pi\left(\frac{a^{2}}{1-n a^{2}}\right)^{n} u u_{\mathrm{t}} r^{2}$.

Where $\mathcal{E}, h, u_{\mathrm{t}}$ and $a$ are the conserved specific energy of the flow excluding its rest mass, specific enthalpy, specific binding energy and local adiabatic sound speed respectively. Equation (5b) may be considered as the outcome of the conservation of mass and entropy along the flow 
line. The expression for $a$ can be written as (Weinberg 1972; Frank et al. 1992):

$a=\left(\frac{\partial p}{\partial \rho}\right)_{\text {Constant Specific Entropy }}^{\frac{1}{2}}=\sqrt{\frac{\gamma p}{\rho}}=\sqrt{\frac{\gamma \kappa T}{\mu m_{\mathrm{H}}}}$.

Where $T$ is the flow temperature, $\mu$ is the mean molecular weight and $m_{\mathrm{H}} \sim m_{\mathrm{p}}$ is the mass of the hydrogen atom. One can now easily derive the expression for the velocity gradient $\left(\frac{\mathrm{d} u}{\mathrm{~d} r}\right)$ (by differentiating Eqs. (5a) and (5b)) as

$\frac{\mathrm{d} u}{\mathrm{~d} r}=\frac{u\left(1-u^{2}\right)\left\{a^{2}(4 r-3)-1\right\}}{2 r(r-1)\left(u^{2}-a^{2}\right)}$.

Since the flow is assumed to be smooth everywhere, if the denominator of Eq. (7a) vanishes at any radial distance $r$, the numerator must also vanish there to maintain the continuity of the flow. One therefore arrives at the so called "sonic point (alternately, the "critical point" ${ }^{1}$ ) conditions" by simultaneously making the numerator and denominator of Eq. (7a) equal zero. The sonic point conditions then can be expressed as follows

$u_{c}=a_{c}=\sqrt{\frac{1}{4 r_{c}-3}}$.

For a specific value of $\mathcal{E}$ and $\gamma$, location of the sonic point $r_{c}^{\mathrm{gr}}$ can be obtained by solving the following equation algebraically

$\mathcal{E}-\frac{(\gamma-1)\left(4 r_{c}-3\right)}{4 r_{c}(\gamma-1)-(3 \gamma+4)} \sqrt{1-\frac{3}{4 r_{c}}}+1=0$.

The spherical surface of radius $r=r_{c}$ can be defined as the "accoustic horizon" because for $r<r_{c}, u>a$ and any accoustic disturbances created in this region are advected towards the black hole. Thus no accoustic disturbances created within this radius can cross the accoustic horizon and escape to the region $r>r_{c}$.

To determine the behaviour of the solution near the sonic point, one needs to evaluate the value of $\left(\frac{\mathrm{d} u}{\mathrm{~d} r}\right)$ at that point (we denote it by $\left(\frac{\mathrm{d} u}{\mathrm{~d} r}\right)_{c}$ ) by applying L'Hospitals' rule to Eq. (7a). It is easy to show that $\left(\frac{\mathrm{d} u}{\mathrm{~d} r}\right)_{c}$ can be obtained by solving the following quadratic equations algebraically:

$$
\begin{aligned}
& \left(\frac{\mathrm{d} u}{\mathrm{~d} r}\right)_{c}^{2}+\frac{(\gamma-1)\left(16 r_{c}^{2}-16 r_{c}-8 \gamma r_{c}+6 \gamma+3\right)}{3 r_{c}\left(4 r_{c}-3\right)^{\frac{3}{2}}}\left(\frac{\mathrm{d} u}{\mathrm{~d} r}\right)_{c} \\
& +\frac{(\gamma-1)\left(2 r_{c}-1\right)\left(24 r_{c}^{2}-28 r_{c}-8 r_{c}^{2} \gamma+4 r_{c} \gamma+3 \gamma+6\right)}{2 r_{c}\left(4 r_{c}-3\right)^{\frac{3}{2}}\left(4 r_{c}-3\right)\left(r_{c}-1\right)} \\
& =0 .
\end{aligned}
$$

It is now quite straightforward to simultaneously solve Eqs. (5a) and (5b) to get the integral curves of the flow for a fixed value of $\mathcal{E}$ and $\gamma$. Detailed methodology for this purpose will be discussed in Sect. 4 .

\footnotetext{
1 Hereafter, we will use "critical ponts" and "sonic points" synonymously.
}

\subsection{Spherical flow in various Pseudo-Schwarzschild space time}

For any "pseudo-Schwarzschild" potential $\Phi_{i}$, the equation of motion for spherically accreting material onto the accretor is given by

$\frac{\partial u}{\partial t}+u \frac{\partial u}{\partial r}+\frac{1}{\rho} \frac{\partial P}{\partial r}+\Phi_{i}^{\prime}=0$

where symbols have their usual meaning. The first term of Eq. (8a) is the Eulerian time derivative of the dynamical velocity at a given $r$, the second term is the "advective" term, the third term is the momentum deposition due to pressure gradient and the final term is due to the gravitational acceleration for a particular $i$ th potential $\Phi_{i}$. The continuity equation can be written as

$\frac{\partial \rho}{\partial t}+\frac{1}{r^{2}} \frac{\partial}{\partial r}\left(\rho u r^{2}\right)=0$.

For a polytropic equation of state, the steady state solution (apart from a geometric factor of $4 \pi$ ) of Eqs. (8a) and $(8 \mathrm{~b})$ is

1) Conservation of specific energy $\mathcal{E}$ of the flow:

$\mathcal{E}=\frac{u^{2}}{2}+\frac{a^{2}}{\gamma-1}+\Phi_{i}$

and

2) Conservation of Baryon number (or accretion rate $\dot{M}_{\text {in }}$ ):

$\dot{M}_{\text {in }}=4 \pi \rho u r^{2}$.

Using $\dot{\mathcal{M}}$ as defined earlier $\left(\dot{\mathcal{M}}=\dot{M}_{\text {in }} \gamma^{n} K^{n}\right)$, Eq. (9b) can be rewritten as

$\dot{\mathcal{M}}=4 \pi a^{2 n} u r^{2}$.

It is now quite straightforward to derive the space gradient of dynamical velocity $\left(\frac{\mathrm{d} u}{\mathrm{~d} r}\right)_{i}$ for flow in any particular $i$ th potential $\Phi_{i}$ as

$\left(\frac{\mathrm{d} u}{\mathrm{~d} r}\right)_{i}=\frac{\frac{2 a^{2}}{r}-\Phi_{i}^{\prime}}{u-\frac{a^{2}}{u}}$.

Using either Eq. (2a) or (2b), one can substitute the value of any specific $i$ th $\Phi_{i}^{\prime}$ in Eq. (10a) to get the value of $\left(\frac{\mathrm{d} u}{\mathrm{~d} r}\right)_{i}$ for motion under particular $i$ th pseudo-Schwarzschild potential. One can also calculate the sonic point quantities (as described in Sect. 3.1) as

$u_{c}^{i}=a_{c}^{i}=\sqrt{\left.\frac{r_{c}^{i}}{2} \Phi_{i}^{\prime}\right|_{c}}$

where superscript $i$ denotes the specific value of sonic quantities for a particular $i$ th potential $\Phi_{i}$, and $\left.\Phi_{i}^{\prime}\right|_{c}$ is the value of $\left(\frac{\mathrm{d} \Phi_{i}}{\mathrm{~d} r}\right)$ evaluated at the corresponding sonic point $r_{c}^{i}$. The value of sonic point $r_{c}^{i}$ for any $i$ th potential $\Phi_{i}$ can be obtained by algebraically solving the following equation

$\mathcal{E}-\left.\frac{1}{2}\left(\frac{\gamma+1}{\gamma-1}\right) r_{c}^{i} \Phi_{i}^{\prime}\right|_{c}-\left.\Phi_{i}\right|_{c}=0$

where $\left.\Phi_{i}\right|_{c}$ is the value of $i$ th potential at the corresponding sonic point $r_{c}^{i}$. Similarly, the value of $\left(\frac{\mathrm{d} u}{\mathrm{~d} r}\right)_{i}$ for any 
$\Phi_{i}$ at its corresponding sonic point $r_{c}^{i}$ can be obtained by solving the following quadratic equation:

$$
\begin{gathered}
(1+\gamma)\left(\frac{\mathrm{d} u}{\mathrm{~d} r}\right)_{c, i}^{2}+2.829(\gamma-1) \sqrt{\frac{\left.\Phi_{i}^{\prime}\right|_{c}}{r_{c}^{i}}}\left(\frac{\mathrm{d} u}{\mathrm{~d} r}\right)_{c, i} \\
+(2 \gamma-1) \frac{\left.\Phi_{i}^{\prime}\right|_{c}}{r_{c}^{i}}+\left.\Phi_{i}^{\prime \prime}\right|_{c}=0
\end{gathered}
$$

where $\left.\Phi_{i}^{\prime \prime}\right|_{c}$ is the value of $\frac{\mathrm{d}^{2} \Phi_{i}}{\mathrm{~d} r^{2}}$ at the corresponding critical point $r_{c}^{i}$.

One can simultaneously solve Eqs. (9a) and (9b) (alternatively, Eqs. (9a) and (9c)) for any specific $\Phi_{i}$ for a fixed value of $\mathcal{E}$ and $\gamma$ to obtain various dynamical and thermodynamic flow quantities. We discuss the detailed methodology in Sect. 4 .

\section{Solution procedure and results}

\subsection{Sonic points as a function of $\mathcal{E}$ and $\gamma$}

Solving Eqs. (7c) and (10c) for a fixed value of $\mathcal{E}$ and $\gamma$, the sonic point could be obtained for FGRSF and for flows in various pseudo-potentials respectively. We denote $r_{c}^{\mathrm{gr}}$ to be the sonic point for FGRSF and $r_{c}^{i}$ to be the sonic point for flow in $i$ th potential $\Phi_{i}$, and define $\Delta r_{c}^{i}$ as:

$\Delta r_{c}^{i}=\frac{r_{c}^{i}-r_{c}^{\mathrm{gr}}}{r_{c}^{\mathrm{gr}}}$

In Fig. 2, we plot various $\Delta r_{c}^{i}$ 's (along the $z$ axis) as a function of specific energy $\mathcal{E}$ (along the $x$ axis) and adiabatic index $\gamma$ (along the $y$ axis) of the flow. The difference in the general profile of variation of $\Delta r_{c}^{i}$ for various $\Phi_{i}$ 's with $\mathcal{E}$ and $\gamma$ is quite obvious from the figure. It is observed that when $\gamma$ is kept fixed at its minimum value $\left(\gamma=\frac{4}{3}\right)$, $\Delta r_{c}^{1}\left(\Delta r_{c}\right.$ for flows in $\left.\Phi_{1}\right)$ is always positive (which means $r_{c}^{1}>r_{c}^{\mathrm{gr}}$ always) and is at a minimum for low energy and starts increasing nonlinearly with an increase of $\mathcal{E}$. At a certain value of $\mathcal{E}$, it produces a peak and starts falling, with further increase of $\mathcal{E}$. The peak value of $\Delta r_{c}^{1}$ is observed to be $\sim 0.15$. Unlike $\Delta r_{c}^{1}, \Delta r_{c}^{2}$ falls very sharply with $\mathcal{E}$ and shows a "dip" at $\mathcal{E} \sim 0.04$, then starts increasing with $\mathcal{E}$ monotonically and nonlinearly, keeping $\Delta r_{c}^{2}$ negative for all values of $\mathcal{E}$ which indicates that $r_{c}^{2}<r_{c}^{\mathrm{gr}}$ for ultra-relativistic flow $\left(\gamma=\frac{4}{3}\right)^{2}$, for all values of $\mathcal{E}$ we consider here. For $\Phi_{3}$ and $\Phi_{4}$, we observe that $\Delta r_{c}^{3}$ and $\Delta r_{c}^{4}$ monotonically decreases with $\mathcal{E}$ and also $\Delta r_{c}^{3}$ and $\Delta r_{c}^{4}$ are always negative e.g., $r_{c}^{3}, r_{c}^{4}<r_{c}^{\mathrm{gr}}$ for all $\mathcal{E}$ we consider here. It is observed that for ultra-relativistic flow, $\Phi_{1}$ is the best approximation to study transonic spherically symmetric polytropic accretion to produce the sonic point closest to the sonic point formed for FGRSF in general, especially in high energy regime, i.e., for high value of $\mathcal{E}$, while $\Phi_{2}$ is the worst approximation for studying

\footnotetext{
${ }^{2}$ Hereafter, we will describe the flow to be ultra-relativistic for $\gamma=\frac{4}{3}$ and purely non-relativistic for $\gamma=\frac{5}{3}$ according to standard practice (Frank et al. 1992).
}

the same phenomena for same set of boundary conditions. If we define a hypothetical quantity $\mathcal{G}_{\mathrm{rc}}^{i}$ which is a measure of "goodness" of a particular $i$ th potential $\Phi_{i}$ regarding the closest approximation of sonic points obtained for FGRFS, we find that following sequence holds good for ultra-relativistic flow:

$\mathcal{G}_{\mathrm{rc}}^{1}>\mathcal{G}_{\mathrm{rc}}^{4}>\mathcal{G}_{\mathrm{rc}}^{3}>\mathcal{G}_{\mathrm{rc}}^{2}$.

The situation starts changing as the value of $\gamma$ increases. The general profile of $\Delta r_{c}^{1}$ is unchanged but its peak starts shifting towards the lower $\mathcal{E}$ values with increasing $\gamma$ and the overall deviation starts increasing. For purely nonrelativistic flow $\left(\gamma=\frac{5}{3}\right),\left.\Delta r_{c}^{1}\right|_{\max }$ is found to be quite large. For all values of $\gamma, \Delta r_{c}^{1}$ is found to be positive, which means that $r_{c}^{1}>r_{c}^{\mathrm{gr}}$ for all values of $\gamma$ and $\mathcal{E}$.

However, the behaviour of $\Delta r_{c}^{2}$ starts changing drastically with an increase in $\gamma$. The "dip" produced by $\Delta r_{c}^{2}$ for low values of $\gamma$ starts smearing out with an increase in $\gamma$ and $\Delta r_{c}^{2}$ becomes positive with higher $\mathcal{E}$ for a fixed value of $\gamma$. For higher $\gamma$ it has been observed that if we study variation of $\Delta r_{c}^{2}$ with $\mathcal{E}$ and $\gamma, \Delta r_{c}^{2}$ starts with a very high negative value at lower $\mathcal{E}$ with $\left|\Delta r_{c}^{2}\right| i_{\max }$ (where $\left|\Delta r_{c}^{2}\right|_{\max }$ stands for the maximum value of the modulus of $\Delta r_{c}^{i}$ ), monotonically decreasing with $\gamma$, reaches zero and becomes positive and increases nonlinearly up to a maximum value (this time, a "peak") and then starts falling again with an increase of $\mathcal{E}$. So for high values of $\gamma$, smearing out of the "dip" in $\Delta r_{c}^{2}$ vs. $\mathcal{E}$ curve is compensated by the appearance of a "peak" at some value of $\mathcal{E}$. It is also observed that the peak starts shifting towards the higher value of $\mathcal{E}$ as $\gamma$ is increased. It is also observed that as we go from ultra-relativistic flow towards purely non-relativistic flow, a zero appears in $\Delta r_{c}^{2}$ vs. $\mathcal{E}$ curve, which indicates that at least one value of $\mathcal{E}$ (for a fixed $\gamma$ ) is available where $r_{c}^{2}$ would be exactly equal to $r_{c}^{\mathrm{gr}}$.

As $\gamma$ is increased, not much change is observed in the general profile of $\Delta r_{c}^{3}$ except that $\left|\Delta r_{c}^{3}\right|_{\max }$ decreases with increase in $\gamma$. The change in profile of $\Delta r_{c}^{4}$ with increasing $\gamma$ is much more interesting. It is observed that with increasing $\gamma$, not only does a zero appear in $\Delta r_{c}^{4}$ vs. $\mathcal{E}$ (for a fixed value of $\gamma$ ) curve (which means that for some values of $\mathcal{E}$ and $\gamma, r_{c}^{4}$ can be exactly equal to $r_{c}^{\mathrm{gr}}$ ), but also the value of $\left|\Delta r_{c}^{4}\right|_{\max }$ decreases considerably with an increase in $\gamma$ and as the flow approaches its purely non-relativistic limit, we observe that the overall deviation produced by $\Phi_{4}$ in approximating $r_{c}^{\mathrm{gr}}$ is quite small in general (percentage deviation produced by $\Phi_{4}$ is found to be within the limit of $10 \%$ for purely non-relativistic flow), which indicates that use of $\Phi_{4}$ is the best possible approximation of the Schwarzschild metric for nonrelativistic flow (flow with high $\gamma$ value), regarding reproduction of the sonic point for a flow with fixed $\mathcal{E}$ and $\gamma$.

\subsection{The integral curves of motion}

We have mentioned earlier that study of the integral curves, i.e., the variation of Mach number of the flow $M$ 

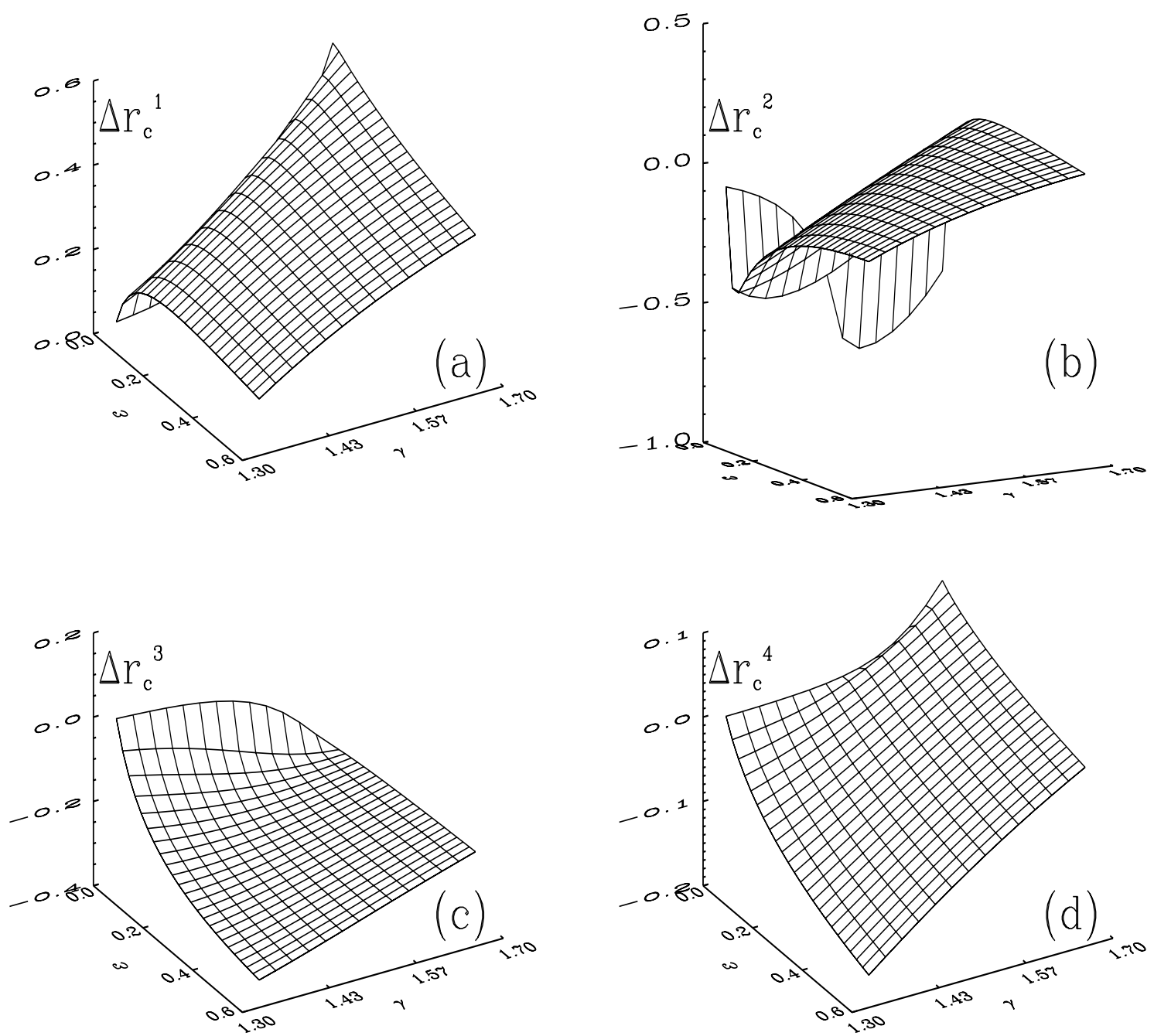

Fig. 2 (a,b,c,d). The deviation $\left(\Delta r_{c}^{i}\right)$ of the value of sonic points $r_{c}^{i}$, s obtained for flows using various $\Phi_{i}$ 's from $r_{c}^{\text {gr }}$ as a function of specific energy $\mathcal{E}$ and adiabatic index $\gamma$ of the flow is plotted for flows in $\Phi_{1}$ (Fig. 1a), $\Phi_{2}$ (Fig. 1b), $\Phi_{3}$ (Fig. 1c) and $\Phi_{4}$ (Fig. 1d). It is observed that while $\Delta r_{c}^{1}$ is a minimum for ultra-relativistic $\left(\gamma=\frac{4}{3}\right)$ flow, $\Delta r_{c}^{4}$ becomes a minimum as the flow approaches a pure-non-relativistic $\left(\gamma=\frac{5}{3}\right)$ limit, see text for detail.

with radial distance $r$ (measured from the accreting hole in units of $r_{\mathrm{g}}$ ) is essential to investigate the "transonicity" of the flow. Let us first consider FGRSF. From Eq. (7c), one can obtain the sonic point $r_{c}^{\mathrm{gr}}$ for a fixed value of $\mathcal{E}$ and $\gamma$. The value of $\left(\frac{\mathrm{d} u}{\mathrm{~d} r}\right)$ at $r_{c}^{\mathrm{gr}}$ is then calculated using Eq. (7d). One can numerically solve Eqs. (5a) and (5b) simultaneously to obtain the value of the Mach number (and other dynamical quantities) as a function of radial distance for a fixed value of $\mathcal{E}$ and $\gamma$. It is well known that for spherically symmetric accretion onto a Schwarzschild black hole, two solutions are obtained while solving the governing conservation equations, e.g., equation for conservation of specific energy and from baryon number (or specific entropy) conservation equation. One solution out of these two corresponds to the accretion process and the other is for the wind. Using Eq. (6), it is easy to calculate the flow temperature at all points of the flow (for accretion as well as for the wind branch).

For accretion in pseudo potentials $\Phi_{i}$ 's, the procedure is exactly the same. Sonic point $r_{c}^{i}$ is obtained by solv- ing Eq. (10c) for the $i$ th potential $\Phi_{i}$ for a fixed value of $\mathcal{E}$ and $\gamma \cdot\left(\frac{\mathrm{d} u}{\mathrm{~d} r}\right)_{c}$ for that particular potential is then obtained by solving Eq. (10d). Starting from $r_{c}^{i}$, Eqs. (9a) and (9b) (alternatively, Eqs. (9a) and (9c)) could be simultaneously solved (using Eq. (10a)) to get the variation of Mach number of the flow (as well as of other dynamical quantities) as a function of radial distance measured in units of $r_{\mathrm{g}}$. The solution for accretion as well as for wind branch can be obtained to investigate the "transonicity" of the flow by plotting Mach number $M$ as a function of radial distance $r$. Temperature of the flow $T^{i}$ (for any $i$ th potential $\Phi_{i}$ ) can easily be obtained by solving Eq. (6) for $a_{i}, p_{i}$ and $\rho_{i}$ for a fixed value of $\mathcal{E}$ and $\gamma$.

In Fig. 3, we plot the variation of Mach number as a function of the radial distance (in logarithmic scale) for FGRSF as well as for flows in all $\Phi_{i}$ 's. The energy of the flow (for FGRSF and for flows in all $\Phi_{i}$ 's) are kept constant at a value 0.0123 and value of $\gamma$ is taken to be $\frac{4}{3}$. It is observed that sonic points of FGRSF $r_{c}^{\mathrm{gr}}$, as well as flows in other potentials $r_{c}^{i}$ are all different, which is 


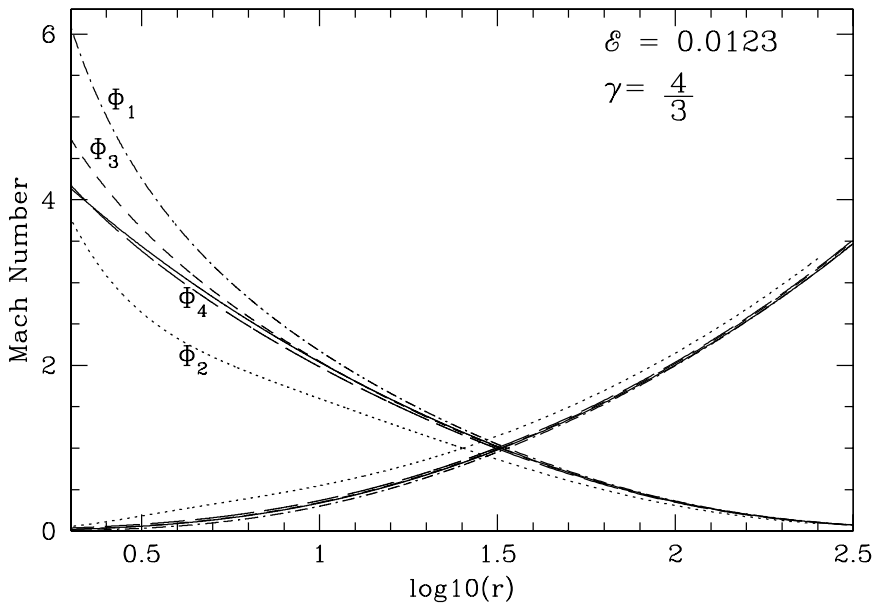

Fig. 3. Variation of Mach number $M$ (plotted along the $Y$ axis) with radial distance (plotted along $X$ axis in logarithmic scale) for accretion and wind for a fixed energy $(\mathcal{E}=0.0123)$ and adiabatic index $\left(\gamma=\frac{4}{3}\right)$ of the flow. The solid lines represent the variation obtained for FGRSF. For same $\mathcal{E}$ and $\gamma$, sonic points $r_{c}^{i}$ 's obtained for flow in defferent pseudo-potentials and for FGRSF are all different which is clearly observed from the figure. While the value of $r_{c}^{\mathrm{gr}}$ is obtained as $32.52 r_{\mathrm{g}}$, values of $r_{c}^{1}, r_{c}^{2}, r_{c}^{3}$ and $r_{c}^{4}$ are obtained as $33.67 r_{\mathrm{g}}, 25.27 r_{\mathrm{g}}, 31.32 r_{\mathrm{g}}$ and $32.12 r_{\mathrm{g}}$ respectively where $r_{c}^{i}$ 's are the sonic points for various $\Phi_{i}$ 's.

normally expected (except for the very special value of $\mathcal{E}$ for which either the $\Delta r_{c}^{2}$ or $\Delta r_{c}^{4}$ vanishes, see Figs. $2 \mathrm{~b}$ and $2 \mathrm{~d}$ ). While the value of $r_{c}^{\mathrm{gr}}$ is obtained as $32.52 r_{\mathrm{g}}$, values of $r_{c}^{1}, r_{c}^{2}, r_{c}^{3}$ and $r_{c}^{4}$ are obtained as $33.67 r_{\mathrm{g}}, 25.27 r_{\mathrm{g}}, 31.32 r_{\mathrm{g}}$ and $32.12 r_{\mathrm{g}}$ respectively. It is also to be noted that although the general profile of the integral curves are almost same (except for flows in $\Phi_{2}$ where the change in curvature is slightly different to that of others, especially for the supersonic accretion branch), the curves do not overlap in general. The amount of deviation (from FGRSF) of integral curves drawn for various $\Phi_{i}$ 's is quite prominent for the supersonic branch of accretion and the derivation is observed to be not that significant (except for flows in $\Phi_{2}$ ) for corresponding subsonic branches of accretion, as well as for the supersonic branch of winds. The deviation maximises with the decrease of radial distance from the accretion black hole. This is quite obvious because close to the accretor, the general relativistic effects would be much more prominent thus the deviation would be higher. Also it is interesting to note that for the same energy $\mathcal{E}$ (and even for a fixed sonic point common to both FGRSF and flows in all $\Phi_{i}$ 's which may be obtained by properly tunning the corresponding $\mathcal{E}$ 's), the maximum value of the Mach number $M_{\max }$ of the flow in different $\Phi_{i}$ 's are very different. One can observe from the figure that there exists a particular value of $r$ (for a fixed $\mathcal{E}$ and $\gamma$ ) where the "crossing-over" of $\Phi_{4}$ with FGRSF at the supersonic branch of the accretion is quite distinct, that is, the Mach number attained for flows in $\Phi_{4}$ at a particular point is exactly equal to the Mach number produced by FGRSF. Also, the general profile for the vari-
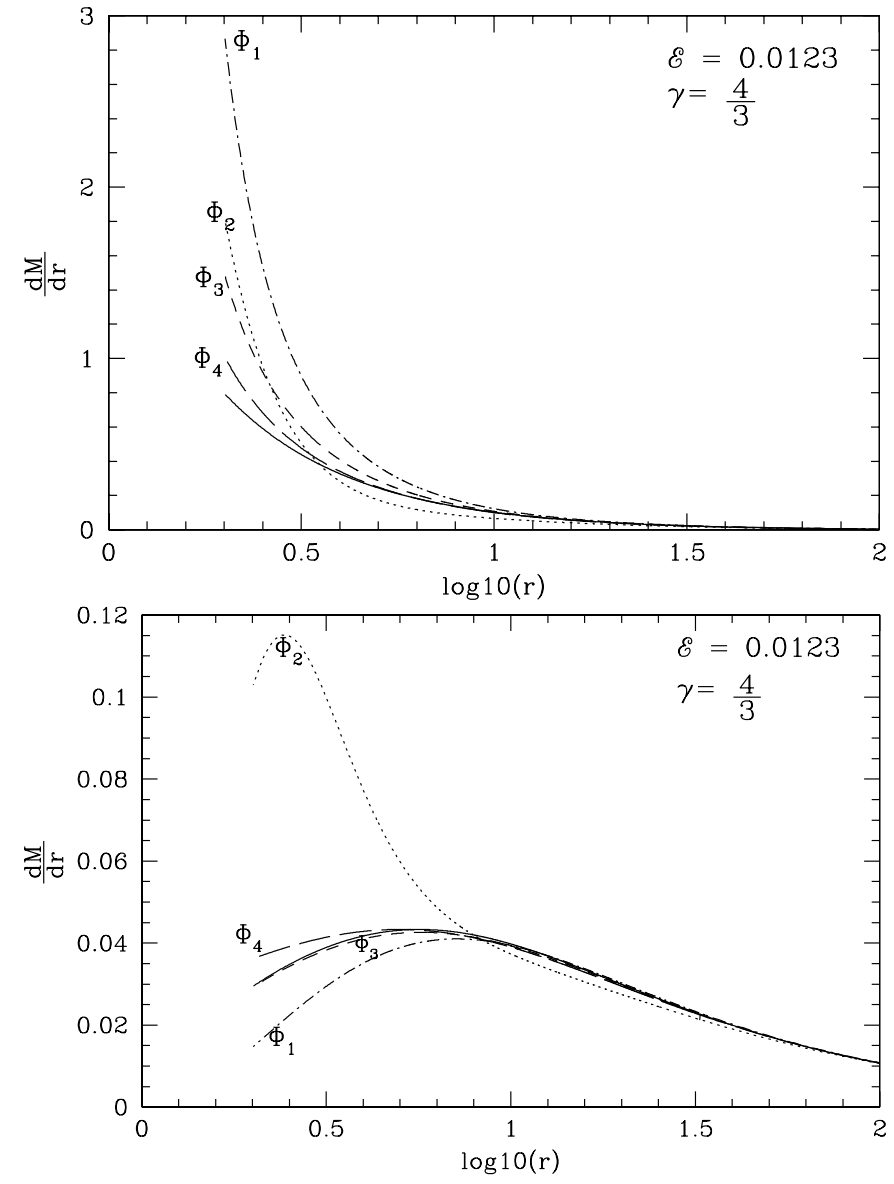

Fig. $4(\mathbf{a}, \mathbf{b})$. Variation of the absolute value of the slope of the Mach number $M$ (plotted on the $Y$ axis as $\frac{\mathrm{d} M}{\mathrm{~d} r}$ ) of accretion (Fig. 4a) and wind (Fig. 4b) with radial distance (plotted along $X$ axis in logarithmic scale) measured from the accretor in units of $r_{\mathrm{g}}$. All input parameters are same as that of Fig. 3. Solid line represents FGRSF. The slope in $M$ is found to increase towards the accretor non-linearly and monotonically for the accretion branch and the space rate of change of $M$ for $\Phi_{1}$ is clearly seen to be steepest while that of $\Phi_{4}$ is observed to be the flattest. For wind, the change is not monotonic; rather it produces a peak at the subsonic branch.

ation of Mach number of the flow in $\Phi_{4}$ is extremely close to the profile obtained in FGRSF almost throughout the flow except very close $\left(r \sim 8 r_{\mathrm{g}}\right.$ in this case) to the black hole. However, no such mutual crossing over is observed in between flows in various $\Phi_{i}$ 's.

If $\Delta T_{\mathrm{ke}}$ and $\Delta T_{\mathrm{th}}$ are the relative gain in the kinetic energy and thermal energy of the flow respectively, it is easy to show that the change in Mach number $\Delta M$ could be approximated as:

$\Delta M \propto \sqrt{\frac{2}{\gamma-1}\left(\frac{\Delta T_{\mathrm{ke}}}{\Delta T_{\mathrm{th}}}\right)}$

for spherical accretion in all pseudo potentials discussed here. Also it is easy to understand that the Mach number profile as well as the rate of change of Mach number at a particular point should be related to the form of the potential $\Phi_{i}$ used to study the flow (and in general to 
the metric used to describe the spacetime). Also $M_{\max }^{i}$, the maximum value of $M$ which could be attended for a particular set of $\mathcal{E}$ and $\gamma$ for flows in any $i$ th potential $\Phi_{i}$ (for the range of $r$ shown here), should be related to the nature of the potential used to describe the flow. It is observed that for $r>2 r_{\mathrm{g}}, M_{\max }^{i}$ anticorrelates with $\mathcal{S}_{i}$ (the "relative stiffness" of the $i$ th potential) for $i=1,3$, 4 but correlates with $\mathcal{S}_{i}$ for $i=2$.

As change in the Mach number is a result of a mutual tug of war in between change in dynamical velocity and polytropic sound speed (alternatively, in between the mechanical and thermal energy of the flow, see Eq. (13)), it might be interesting to investigate the variation of the slope of the Mach number (which might be considered as the measure of the degree of "transonicity" of the flow) with radial distance $r$ for two different branches of solutions, namely, accretion and wind. In Figs. $4 \mathrm{a}$ and $4 \mathrm{~b}$ we plot the absolute value of $\left(\frac{\mathrm{d} M}{\mathrm{~d} r}\right)$ as a function of $\log 10(r)$ for accretion (Fig. 4a) and wind (Fig. 4b) for FGRSF as well as for flows in various $\Phi_{i}$ s. For accretion we see that $\left|\frac{\mathrm{d} M}{\mathrm{~d} r}\right|$ increases nonlinearly and monotonically as the flow comes closer to the black hole, which indicates that for all flows, instantaneous changes in kinetic energy of the flow is always greater than the instantaneous change in thermal energy, i.e., $\Delta T_{\mathrm{ke}}>\Delta T_{\mathrm{th}}$ for all values of $r$, which is not the case for winds. For winds (see Fig. 4b), change of $\left|\frac{\mathrm{d} M}{\mathrm{~d} r}\right|$ is not monotonic rather it always shows a peak in the subsonic branch for FGRSF as well as for flows in all $\Phi_{i}$ 's. The appearance of the peak for flows in pseudo potentials is due to the fact that from the close vicinity of the event horizon up to a certain distance $r_{\mathrm{p}}\left(r_{\mathrm{p}}^{i}<r_{c}^{i}\right.$ and $r_{\mathrm{p}}^{\mathrm{gr}}<r_{c}^{\mathrm{gr}}$ always), the local gain in kinetic energy at any point is higher than the local thermal energy gain. So up to $r_{\mathrm{p}}, \Delta T_{\mathrm{ke}}>\Delta T_{\mathrm{th}}$ but after $r_{\mathrm{p}}, \Delta T_{\mathrm{ke}}$ decreases and $\left|\frac{\mathrm{d} M}{\mathrm{~d} r}\right|$ starts falling as the wind approaches to the sonic point. It is easy to understand that as $\mathcal{E}$ is kept constant throughout the flow (for accretion as well as for the wind branch), the bulk motion acceleration of the flow is a continuous process throughout the accretion towards the black hole but for winds the acceleration process dominates only up to $r_{\mathrm{p}}$ and gets the major part of the outward acceleration in the region bounded in $r_{\mathrm{g}}>r>r_{\mathrm{p}}^{i}$, though the exact physical reason behind this is not clearly understood. However, it should be remembered that the above argument holds only for flows in pseudo potentials and not for FGRSF; because for FGRSF, the total energy term can not be decoupled into various counterparts with individual origin e.g., mechanical energy $\mathcal{E}_{\mathrm{ke}}=\frac{u^{2}}{2}$ or thermal energy $\mathcal{E}_{\text {th }}=\frac{a^{2}}{\gamma-1}$ etc.

It is clear from the figure that the location of $r_{\mathrm{p}}^{1}\left(r_{\mathrm{p}}\right.$ for flows in $\Phi_{1}$ ) is located furthest away from the black hole and $r_{\mathrm{p}}^{2}$ is located closest to the black hole with the following sequence:

$r_{\mathrm{p}}^{1}<r_{\mathrm{p}}^{3}<r_{\mathrm{p}}^{\mathrm{gr}}<r_{\mathrm{p}}^{4}<r_{\mathrm{p}}^{2}$.

It is also observed that with increase of $\gamma$, the sequence in Eq. (14) is maintained but the location of all $r_{\mathrm{p}} \mathrm{s}$ moves

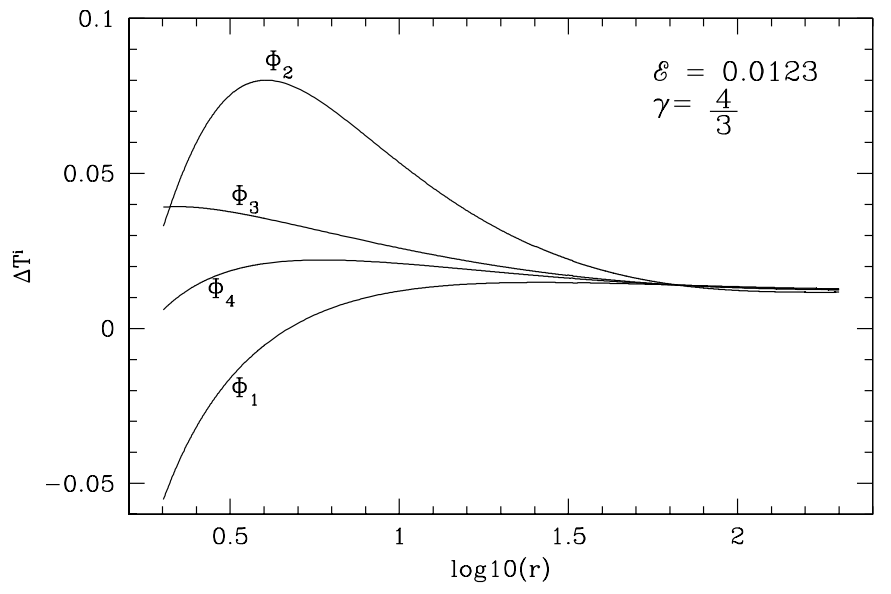

Fig. 5. Variation of the temperature deviation $\left(\Delta T^{i} \mathrm{~s}\right)$ of the accretion flow with radial distance for ultra relativistic $\left(\gamma=\frac{4}{3}\right)$ flow in various $\Phi_{i}$ s. While the difference in flow temperature in between any $T^{i}$ (flow temperature for a particular $i$ th potential $\Phi_{i}$ ) and $T^{\mathrm{gr}}$ (flow temperature for FGRSF), is plotted along the $Y$ axis, the distance from the central accretor (in logarithmic scale in units of $r_{\mathrm{g}}$ ) is plotted along $X$ axis. The maximum amount of deviation is obtained close to the accretor, which is expected. It is clearly observed from the figure that use of $\Phi_{4}$ and (except very close to the black hole) $\Phi_{1}$ are the best approximation of the FGRSF flow temperature. Almost the same sort of deviation is found for the wind branch (not shown in the figure).

towards the black hole. Thus, the regions from where the wind (in various $\Phi_{i}$ 's) is accelerated, moves closer to the hole as the flow approaches to its non-relativistic limit. If we define $\Delta\left(\frac{\mathrm{d} M}{\mathrm{~d} r}\right)^{i}$ to be:

$\Delta\left(\frac{\mathrm{d} M}{\mathrm{~d} r}\right)^{i}=\frac{\left(\frac{\mathrm{d} M}{\mathrm{~d} r}\right)^{i}-\left(\frac{\mathrm{d} M}{\mathrm{~d} r}\right)^{\mathrm{gr}}}{\left(\frac{\mathrm{d} M}{\mathrm{~d} r}\right)^{\mathrm{gr}}}$

then one can show that for a fixed value of $\mathcal{E}$ and $\gamma$, the following sequence are maintained for various $\Phi_{i}$ s as:

$$
\begin{aligned}
\left|\Delta\left(\frac{\mathrm{d} M}{\mathrm{~d} r}\right)^{3}\right|_{\max } & <\left|\Delta\left(\frac{\mathrm{d} M}{\mathrm{~d} r}\right)^{4}\right|_{\max }<\left|\Delta\left(\frac{\mathrm{d} M}{\mathrm{~d} r}\right)^{2}\right|_{\max } \\
& <\left|\Delta\left(\frac{\mathrm{d} M}{\mathrm{~d} r}\right)^{1}\right|_{\max }
\end{aligned}
$$

for accretion where $\left|\Delta\left(\frac{\mathrm{d} M}{\mathrm{~d} r}\right)^{i}\right|_{\max }$ stands for the maximum value of $\Delta\left(\frac{\mathrm{d} M}{\mathrm{~d} r}\right)^{i}$. Whereas for wind it is observed that:

$$
\begin{aligned}
\left|\Delta\left(\frac{\mathrm{d} M}{\mathrm{~d} r}\right)^{4}\right|_{\max } & <\left|\Delta\left(\frac{\mathrm{d} M}{\mathrm{~d} r}\right)^{3}\right|_{\max }<\left|\Delta\left(\frac{\mathrm{d} M}{\mathrm{~d} r}\right)^{1}\right|_{\max } \\
& <\left|\Delta\left(\frac{\mathrm{d} M}{\mathrm{~d} r}\right)^{2}\right|_{\max } .
\end{aligned}
$$

Also note that for the accretion branch all $\Delta\left(\frac{\mathrm{d} M}{\mathrm{~d} r}\right)^{i}$ change sign except $\Delta\left(\frac{\mathrm{d} M}{\mathrm{~d} r}\right)^{1}$. 


\subsection{Temperature profile of the flow}

One can easily show that at any point of the flow, the flow temp $T^{i}$ for flow in any particular $\Phi_{i}$ s can be re-written as:

$T^{i}=\frac{\mu m_{\mathrm{p}}}{\gamma \kappa}\left\{\frac{(2 \gamma-1)\left(\mathcal{E}-\Phi_{i}\right)}{2+M^{i}(\gamma-1)}\right\}$

where $M^{i}$ is the corresponding Mach number for the $i$ th pseudo potential. As it has been stated that $M^{i}$ can be calculated at each point of the flow, one can compute $T^{i}$ from knowledge of $M^{i}$ s using Eq. (16). After defining $\Delta T^{i}$ to be:

$\Delta T^{i}=\frac{T^{i}-T^{\mathrm{gr}}}{T^{\mathrm{gr}}}$

where $T^{\mathrm{gr}}$ could be computed using Eq. (6), one can plot $\Delta T^{i}$ as a function of radial distance (in units of $r_{\mathrm{g}}$ ) to investigate which $\Phi_{i}$ produces the corresponding $T^{i}$ closest to $T^{\mathrm{gr}}$ at a particular radial distance and for a fixed value of the $\mathcal{E}$ and $\gamma$. In Fig. 5 we plot $\Delta T^{i}$ vs. $\log 10(r)$ to demonstrate the dependence of $\Delta T^{i}$ on radial distance. It is clear from the figure that except for $T^{1}$, all other $T^{i} \mathrm{~S}$ are always higher than $T^{\mathrm{gr}}$, which indicates that all $\Phi_{i} \mathrm{~S}$ except $\Phi_{1}$ produce hotter flow than FGRSF in general. For $\Phi_{1}$, very close to the hole the flow is cooler compared to FGRSF up to a certain distance, after which $\Phi_{1}$ also produces $T^{1}$ higher to $T^{\mathrm{gr}}$. The point at which $T^{1}$ becomes equal to $T^{\mathrm{gr}}$, gets shifted away from the black hole with increasing $\gamma$, keeping $\mathcal{E}$ fixed. Also, it is observed that $\left|\Delta T^{i}\right|_{\max }$ increases with increasing $\gamma$. From Fig. 5 it is clear that throughout its way from infinity to the event horizon, $\Phi_{1}$ and $\Phi_{4}$ produces very good approximation of FGRSF regarding calculation of flow temperature. However, after a certain point very close to the black hole (after the crossing the point where $T^{1}=T^{\mathrm{gr}}$ ), $\Phi_{1}$ produces a sudden and sharp deviation of the flow temperature from FGRSF, physical reason for which is not clearly understood. More or less the same sort of profile in the $\Delta T^{i}$ vs. $\log 10(r)$ curve is also observed for wind branch.

\section{Conclusion}

In this paper we have solved a set of algebraic and differential equations governing various dynamical and thermodynamic behaviouars of Bondi (1954) type accretion in a full Schwarzschild metric as well as for motion under a number of "pseudo-Schwarzschild" potentials, to examine the suitability in application of those potentials in investigating spherically symmetric transonic accretion onto a nonrotating black hole. We have shown that though the potentials discussed here were originally proposed to mimic the relativistic effects manifested in the disc accretion, it is quite reasonable to use most of the potentials in studying various dynamical as well as thermodynamic quantities for spherical accretion. Also, we have shown that depending on the chosen regions of parameter space spanned by specific energy $\mathcal{E}$ and adiabatic index $\gamma$ of the flow, one potential may be important than others and we could identify which potential is the best approximation for FGRSF for what values of $\mathcal{E}$ and $\gamma$. We have restricted ourselves to the study of simple polytropic flows only. However, the validity of using all these $\Phi_{i}$ s discussed here can easily be examined for isothermal accretion and wind as well as for flows with other equations of state. Work in this direction is reported elsewhere (Sarkar \& Das 2001).

It is observed that among all pseudo potentials, $\Phi_{1}$ (potential proposed by Paczyński \& Wiita 1980) and $\Phi_{4}$ (one of the potentials proposed by Artemova et al. 1996) are in general the best in the sense that they provide very reasonable approximation to the full general relativistic solution. While $\Phi_{1}$ is the best approximation for ultrarelativistic flow, $\Phi_{4}$ happens to be the best approximation as the flow tends to be fully non relativistic, i.e, $\gamma$ tends to have the value $\frac{5}{3}$. Also we see that there are certain cases for which one or more of the pseudo potentials may give the exact match with FGRSF for a particular value of $\mathcal{E}$ or $\gamma$ (for a fixed $r$ ) in finding some dynamical $\left(r_{c}, M\right.$ etc.) or thermodynamic (flow temperature $T$, for example) quantity.

It is worth mentioning that as long as one is not interested in astrophysical processes extremely close (within $1-2 r_{\mathrm{g}}$ ) to a black hole horizon, one may safely use the "pseudo" potentials discussed here to study spherically symmetric accretion on to a Schwarzschild black hole with the advantage that use of these potentials would simplify calculations by allowing one to use some basic features of flat geometry (additivity of energy or de-coupling of various energy components, i.e., thermal $\left(\frac{a^{2}}{\gamma-1}\right)$ Kinetic $\left(\frac{u^{2}}{2}\right)$ or gravitational $(\Phi)$ etc.) which is not possible for calculations in a purely Schawarzschild metric. Also, one can study more complex many body problems such as accretion from an ensemble of companions or overall effeciency of accretion onto an ensemble of black holes in a galaxy or for studying numerical hydrodynamic or magnetohydrodynamic flows around a black hole etc. as simply as can be done in a Newtonian framework, but with far better accuracy. However, one should be careful in using these potentials to study spherically symmetric accretion because of the fact that none of the potentials discussed here are "exact" in a sense that they are not directly derivable from the Einstein equations. These potentials could only be used to obtain more accurate correction terms over and above the pure Newtonian results and any "radically" new results obtained using these potentials should be crosschecked very carefully with the exact general relativistic theory.

Although the theory of disc accretion has priority over spherical accretion because of the fact that accretion discs describe more realistic situations found in nature, it is not unreasonable to concentrate on spherical accretion because for certain cases, that may be quite useful and use of these potentials makes a complicated problem simpler to study. For example, for a supermassive black hole immersed in intergalactic space in such a way that matter falling on to it has negligible intrinsic angular momentum, 
the accretion (at least close to the hole) is quasi spherical and transonic spherical accretion might be a good approximation to mimic the situation. Same sort of approximation is valid when an accreting black hole is embedded in a number of donor stars (or star clusters) where the angular momentum of the stars are randomly oriented in such a way that the vector sum of the intrinsic angular momentum carried by the accreting matter as a whole may be quite negligible, so as to make Bondi-type accretion a good approximation. In fact, a number of recent works (Coker \& Markoff 2001 and references therein; Das 1999, 2000, 2001a,b; Toropin et al. 1999; Kovalenko \& Eremin 1998; Titarchuk et al. 1996, 1997; Wang \& Sutherland 1997; Zampieri et al. 1996; Yim \& Park 1995; Markovic 1995; Tsuribe et al. 1995; Kazhdan \& Murzina 1994; Fortner 1993) still deal with spherical accretion to investigate some basic astrophysical processes the black holes and neutron stars. So we believe that work presented in this paper is relevant and will be useful in investigation of various aspects of accretion and wind around non-rotating and slowly rotating compact objects.

Acknowledgements. We are grateful to Prof. P. J. Wiita and Prof. I. Novikov for useful discussions.

\section{References}

Artemova, I. V., Bjornsson, G., \& Novikov, I. D. 1996, ApJ, 461, 565

Begelman, M. C. 1978, A\&A, 70, 583

Blumenthal, G. R., \& Mathews, W. G. 1976, ApJ, 203, 714

Bondi, H. 1952, MNRAS, 112, 195

Brinkmann, W. 1980, A\&A, 85, 146

Chakrabarti, S. K. 1996, Phys. Reports, 266, No. 5 \& 6, 229

Coker, R. F., \& Markoff, S. 2001, to appear in the Proceedings of IAU, 205

Das, T. K. 1999, MNRAS, 308, 201

Das, T. K. 2000, MNRAS, 318, 294
Das, T. K. 2001a, submitted to MNRAS

Das, T. K. 2001b, A\&A, in press

Frank, J., King, A., \& Raine, D. 1992, Accretion Power in Astrophysics, 2nd edition (Cambridge University Press)

Fortner, B. I. 1993, Ph.D. Thesis, Illinois Univ., UrbanaChampaign

Kazhdan, Y. M., \& Murzina, M. 1994, MNRAS, 270, 351

Kovalenko, I. G., \& Eremin, M. A. 1998, MNRAS, 298, 861

Malec, E. 1999, Phys. Rev. D, 60, 104043

Markovic, D. 1995, MNRAS, 277, 11

Michel, F. C. 1972, Astrophys. Space Sci., 15, 153

Novikov, I. D., \& Thorne, K. 1973, in Black Holes, Les Astres Occlus, ed. B \& C. De Witt (New York: Gordon \& Breach), 343

Nowak, A. M., \& Wagoner, R. V. 1991, ApJ, 378, 656

Paczyński, B., \& Wiita, P. J. 1980, A\&A, 88, 23

Riffert, H., \& Herold, H. 1995, ApJ, 450, 508

Shapiro, S. L., \& Teukolsky, S. A. 1983, Black Holes, White Dwarfs and Neutrin stars (John Wiley and Sons Inc)

Sarkar, A., \& Das, T. K. 2001, IJMPD, in press

Shakura, N. I., \& Sunyaev, R. A. 1973, A\&A, 24, 337

Titarchuk, L., Mastichiadis, A., \& Kylafis, N. D. 1997, ApJ, 487,834

Titarchuk, L., Mastichiadis, A., \& Kylafis, N. D. 1996, A\&A, 120, 171

Toropin, Yu. M., Toropina, O. D., Savelyev, V. V., Romanova, M. M., Chechetkin, V. M., \& Lovelace, R. V. E. 1999, ApJ, 517,906

Wang, J. C. L., \& Sutherland, R. S. 1998, in Accretion Processes in Astrophysical Systems: Some Like it Hot! ed. S. S. Holt, \& T. R. Kallman, AIP Conf. Proc., 431, 413

Tsuribe, T., Umemura, M., \& Fukue, J. 1995, Publications of the Astronomical Society of Japan, vol. 47, No. 1, 73

Weinberg, S. 1972, Gravitation and Cosmology: Principles and Applications of the General Theory of Relativity (John Wiley \& Sons)

Yim, S. Y., \& Park, M. 1995, Publications of the Korean Astronomical Society, 10(1), 79

Zampieri, L., Miller, J. C., \& Turolla, R. 1996, MNRAS, 281, 1183 